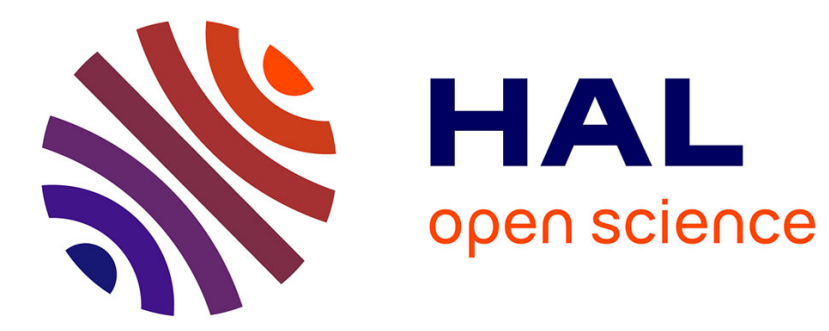

\title{
La discipline de l'ivresse dans une confrérie musulmane de Turquie
}

\author{
Nicolas Elias
}

\section{To cite this version:}

Nicolas Elias. La discipline de l'ivresse dans une confrérie musulmane de Turquie. Archives de Sciences Sociales des Religions, 2016, 174, pp.241-254. 10.4000/assr.27818 . hal-03177158

\section{HAL Id: hal-03177158 \\ https://hal.science/hal-03177158}

Submitted on 3 Jun 2021

HAL is a multi-disciplinary open access archive for the deposit and dissemination of scientific research documents, whether they are published or not. The documents may come from teaching and research institutions in France or abroad, or from public or private research centers.
L'archive ouverte pluridisciplinaire HAL, est destinée au dépôt et à la diffusion de documents scientifiques de niveau recherche, publiés ou non, émanant des établissements d'enseignement et de recherche français ou étrangers, des laboratoires publics ou privés. 


\section{Archives de sciences sociales des religions}

174 | Avril-Juin 2016

La force des objets - Matières à expériences

\section{La discipline de l'ivresse dans une confrérie musulmane de Turquie}

The discipline of drunkenness in a Muslim brotherhood of Turkey

La disciplina de la embriaguez en una cofradía musulmana de Turquía

\section{Nicolas Elias}

\section{CpenEdition}

Journals

Édition électronique

URL : http://journals.openedition.org/assr/27818

DOI : $10.4000 /$ assr. 27818

ISSN : $1777-5825$

Éditeur

Éditions de l'EHESS

Édition imprimée

Date de publication : 1 avril 2016

Pagination : 241-254

ISBN : 978-2-7132-25-17-8

ISSN : 0335-5985

\section{Référence électronique}

Nicolas Elias, "La discipline de l'ivresse dans une confrérie musulmane de Turquie », Archives de sciences sociales des religions [En ligne], 174 | Avril-Juin 2016, mis en ligne le 01 avril 2018, consulté le 10 décembre 2020. URL : http://journals.openedition.org/assr/27818 ; DOI : https://doi.org/10.4000/ assr.27818 
La vie de la matière 



\section{Nicolas Elias}

\section{La discipline de l'ivresse dans une confrérie musulmane de Turquie}

«Sans alcool, il n'y a pas de liturgie possible» (dem yoksa ibadet olmaz). De la part d'un dignitaire bektaşi, une confrérie majeure de l'islam anatolien et balkanique, ces propos ont tout l'air d'une provocation. Que ceux qui se réclament de l'autorité de Hacı Bektaş Veli, «saint » de l'Anatolie médiévale qui donna son nom à la confrérie bektaşi, prennent des libertés avec les interdits religieux est, en Turquie, de notoriété publique. Ils sont accusés, généralement à raison, de ne pas se joindre aux prières à la mosquée, de ne pas observer le jeûne du ramadan ou encore de consommer de l'alcool malgré l'interdit (haram). De fait, la confrérie professe une compréhension ésotérique de l'islam, dans laquelle le Coran est remplacé par les enseignements de maîtres passés sous la forme de poèmes ou d'hagiographies (vilayetname) en langue turque. Mais que certains d'entre eux aillent jusqu'à boire durant la cérémonie, jusqu'à faire de la transgression un acte de piété, voilà qui est plus intrigant, surtout lorsque l'on sait l'hypersensibilité qui entoure l'alcool en Turquie ${ }^{1}$. Force est pourtant de constater que cette phrase résume parfaitement l'opinion et les pratiques des villageois de Tekke Köyü (littéralement « le village du couvent »), village d'initiés bektaşi, aujourd'hui relativement isolé dans les montagnes du Taurus mais héritier de ce qui fut l'un des principaux couvent de la confrérie (Köprülü, 2001). Lors des cérémonies, du rakı (un alcool de raisin anisé, obtenu par distillation) est consommé en quantité trop importante pour n'être qu'un symbole communiel, mais sans pour autant donner lieu à aucun débordement. En effet, une stricte discipline est de mise parmi les participants.

Le sujet est éminemment délicat, d'autant qu'il y a là de quoi nourrir les calomnies dont les bektaşi ont fait l'objet par le passé (celles de s'adonner, dans ces réunions secrètes et mixtes, à la débauche). Le village de Tekke revendique

1. Ainsi en juin 2013, au plus fort des contestations anti-gouvernementales, et alors que le nombre de morts allait croissant, la question de savoir si oui ou non des manifestants avaient consommé de la bière dans la mosquée de Dolmabahçe où ils s’étaient réfugiés avait agité une bonne partie de la presse et du pays (la réponse était non). 
d'ailleurs un rite (erkan) singulier au sein de cet archipel complexe qu'est aujourd'hui la confrérie bektaşi ${ }^{2}$. Cependant, ces cérémonies villageoises peuvent très certainement permettre de repenser efficacement, au-delà des préjugés ou de la simple transgression, une pratique à la fois fortement décriée par ses détracteurs et dissimulée par les intéressés : un usage liturgique de l'alcool dans une confrérie musulmane, ou comment un travail du sens de l'ivresse peut participer de l'instauration de l'ordre confrérique, comme de l'initiation de ses membres.

Tekke Köyü est un village relativement opulent, riche de vergers, de troupeaux de mouton, et d'une vie confrérique foisonnante : la très grande majorité des adultes sont initiés - en couple, après leur mariage -, et, si la belle saison est dévolue aux travaux des champs comme à la transhumance dans les alpages, ce sont les longues cérémonies du cem qui occupent les villageois tout l'hiver. Ces cérémonies "de l'union " (sens du mot cem), qui se substituent aux prières collectives à la mosquée des sunnites, sont au cœur de la vie villageoise : non seulement y prennent place les temps forts de la socialité confrérique - initiation, règlement des conflits ${ }^{3}$, commensalité rituelle - et le rappel de sa généalogie spirituelle - évocation des figures tutélaires, invocation aux douze imams, commémoration du drame de Kerbela -, mais l'organisation et la succession de ces cérémonies durant l'hiver repose sur un large système d'offrandes mutuelles et partagées. Ainsi, chacun doit-il participer à l'offrande d'un cem, une dépense financière substantielle ${ }^{4}$, à la communauté dans l'année liturgique, et ce, selon l'ordre de prééminence dans la hiérarchie confrérique. Commençant à la nuit tombée, et finissant rarement avant minuit, chaque cérémonie est placée sous l'autorité d'un baba (littéralement le "père ») réélu chaque année, et son accès est en théorie réservé aux initiés de l'ordre confrérique. Une culture du secret entoure sa tenue. Ce secret ou "dissimulation»(takiyye), qui est aujourd'hui constitutif de la confrérie, n'est nullement accessoire : conjuguant musique, qui plus est musique instrumentale, danse, mixité sexuelle, et, ici, consommation rituelle d'alcool, le cem a de quoi choquer les plus rigoristes.

\section{Instituer l'ivresse}

Je n'évoquerai pas l'ensemble de la cérémonie, pour me focaliser sur sa phase la plus inconvenante selon les normes du sunnisme, celle qui concentre la totalité

2. Les initiés du village affirment pratiquer le rite dit « de Kaygusuz " (kaygusuz erkan ), quand la majeure partie de la confrérie et ses plus hautes autorités suivent celui dit « de Balım Sultan " (balım Sultan erkanı). Cette singularité, légitimée par l'importance historique du lieu, n'implique cependant aucune rupture, et les dignitaires de la congrégation villageoise ont reçu l'investiture (icazet) des autorités du rite principal.

3. Réduit ici à une question rhétorique.

4. L'organisation d'un cem coûterait approximativement 1500-2 000 livres turques. 
de la consommation d'alcool, et que l'on nomme mubabbet ${ }^{5}$. Séquence médiane, enchâssée entre deux phases plus formelles, le muhabbet est le temps du ventre et des oreilles ${ }^{6}$. Les participants se regroupent par quatre ou cinq autour de larges plateaux de cuivre (sofra) pour un copieux repas, entrecoupé par les sermons du baba qui rompent avec le caractère formel des prières et des chants " assis " (nommés ainsi par opposition aux airs de danse). Cette séquence se clôt par une danse collective, et donc mixte, la "danse des Quarante ( kırklar semahı), transition vers la séquence conclusive de la cérémonie. Cette courte description n'est pas propice à donner une haute idée de ce temps liturgique, qui pourrait ainsi être assimilé à un acte trivial. Il est pourtant fondamental, aux dires même d'un baba, qui explique qu' " on ne s'approche pas d'Allah avec le rite ou la répétition des noms divins $(z i k r)$ mais grâce au mubabbet ».

Il y aurait quelques mots à dire sur les textes chantés. Ces courts poèmes en langue turque, accompagnés par un luth, portent une bonne partie du sens ésotérique propre à la confrérie. Un certain nombre d'entre eux touche d'ailleurs à l'alcool. Il y aurait plus encore à dire sur le parallèle entre le mubabbet et la légende des Quarante qui, quoiqu'occultée à Tekke Köyü, constitue une sorte de soubassement narratif de la cérémonie du cem. Brodée sur la tradition islamique de l'ascension de Muhammed (miraç), elle met en scène la rencontre entre celui-ci et l'assemblée des Quarante (dont l'identité n'est pas claire) présidée par son gendre Ali. S'ensuit un dialogue énigmatique, établissant la préséance spirituelle d'Ali sur le prophète, qui se clôt par l'absorption du jus d'un unique grain de raisin par l'assemblée, laquelle, rendue ivre, se met à danser le semah, le semah « des Quarante» justement (Mélikoff, 1998 : 206).

De fait, l'alcool tient une place fondamentale dans le muhabbet, objet d'une attention toute particulière. Si le «raisin » est à Tekke Köyü consommé sous forme de rakı (distillation à environ $45 \%$ d'alcool), il n'est jamais mentionné comme tel, ni même comme alcool (içki), mais toujours sous le vocable de dem, un mot riche de sens qui se nourrit d'une double étymologie - arabe et persane et signifie tout à la fois le "souffle ", l' «instant» (acception persane), et le «sang » (acception arabe). À la fois donc le fugace, l'inconsistant, et le fondamental, le concentré ${ }^{7}$. D'autre part, sa mise en circulation et son absorption suivent un protocole élaboré, que l'on pourrait résumer selon deux principes.

5. Si l'étymologie arabe du terme renvoie à la notion d'amour, il désigne plus prosaïquement en turc actuel un entretien amical, un temps de convivialité (souvent musical).

6. L'articulation en trois phases, et cette position médiane du mubabbet, semblent spécifiques au rite de Kaygusuz. Birge (1937) et Soileau (2012), décrivant le rite de Balım Sultan, respectivement à Istanbul et à Ankara, observent une disjonction entre l'office proprement dit et le banquet (alcoolisé) qui lui succède, soit une articulation en deux phases. Placer la consommation d'alcool comme relevant du paraliturgique pourrait être un procédé de légitimation ou de justification, quoique Birge reste évasif sur la présence ou non d'alcool lors de l'office proprement dit (cf. Birge, 1937 : 196). Notons qu'à Tekke Köyü, un mubabbet organisé hors du cem sera désigné comme "muhabbet de Balım " (balım muhabbeti).

7. Dem renvoie principalement, pour les profanes, au thé fortement infusé du samovar, que l'on coupe d'eau bouillante. 
Le premier de ces principes est que les participants n'ont pas un accès direct à l'alcool, que celui-ci est médiatisé par un officiant, le saki, terme que l'on traduit généralement en français par "échanson ", et qui veut dire plus simplement "préposé à la boisson ». Ce dernier mélange dans une grande bassine en plastique le contenu de plusieurs bouteilles achetées dans le commerce avec une égale quantité d'eau, puis répartit le tout entre les différents groupes dans des bassines de plus petite taille. À chaque «table ", un convive aura la charge de saki délégué. Lui seul aura accès à la bassine de dem : il y plongera la coupe unique qu'il tendra successivement à chacun. De la bassine aux lèvres, la procédure n'est pas moins contraignante, ni moins signifiante. La coupe est toujours tenue des deux mains, ou plutôt dissimulée entre les deux mains, de sorte que l'on ne voit pas son contenu. "Que l'amour soit " (aşk olsun) dira celui qui la tend, à quoi celui qui la reçoit répondra : "Que ton amour soit resplendissant " (aşkın cemal olsun).

Je dis « les participants ", mais ce sont principalement les hommes qui boivent. S'il ne semble pas y avoir de tabou concernant la consommation féminine, et si certaines parmi les plus âgées affirment avoir déjà bu, les femmes, qui se tiennent prioritairement au fond de la pièce (sans qu'il y ait, là encore, une séparation stricte $)^{8}$, ne boivent généralement pas.

Quant au second principe, régissant lui la consommation, il a été parfaitement énoncé par Mark Soileau, qui a commenté les pratiques du sofra dans une communauté bektaşi d'Ankara : "Personne ne prend une gorgée de dem avant le signal du $b a b a$, et toujours à la suite d'une gorgée bue par le baba lui-même » (Soileau, 2012 : 18). Comme la précédente, cette règle ne souffre aucune exception. Les premières gorgées sont bues selon la coutume du üçleme, ce qu'Irène Mélikoff traduit par "trisser» (1998: 209), trois gorgées ouvrant le temps de l'alcool et bues en dévotion à Allah, Muhammed et Ali, la trinité de la confrérie. "Pour l'amour des Trois» (üçler aşkına) dira alors le baba. Puis, à intervalles réguliers, et à des moments qu'il juge signifiants durant les sermons et les poèmes chantés, il enjoindra à l'assemblée de boire. La prise d'alcool intervient comme pour souligner les paroles. Là encore, Mark Soileau interprète très justement cette ponctuation dans le déroulement du muhabbet, et plus spécifiquement celle des mots par l'alcool : le dem est marqueur de l'instant (l'une des significations du mot dem, on l'a dit) et ingurgitation du sens (Soileau, 2012:25).

L'ivresse est ici une technique liturgique du corps. C'est bien là le sens de la phrase placée en introduction de ce texte: "sans dem, il n'y a pas de liturgie possible». Son exercice imprime une temporalité propre, celle d'une lente et progressive modification de l'état de conscience, qui est vécue comme l'imprégnation d'un sens initiatique. L'ivresse comme accès vers le divin est l'un des topö̈ de la littérature bektaşi, et c'est en ce sens qu'il faut entendre la déclaration citée

8. La relative mixité sexuelle est à appréhender dans un vis-à-vis avec la stricte ségrégation en usage dans les mosquées. 
précédemment selon laquelle on se rapproche d'Allah par le mubabbet. John Kingsley Birge, qui publie en 1937 l'une des études les plus complètes sur l'ordre (Birge, 1937), n'hésite pas à comparer la prise d'alcool à d'autres techniques corporelles "soufies » mieux connues, le $z i k r$ des «derviches hurleurs " et le sema des "derviches tourneurs ", qui visent tous deux, par des mouvements du corps et des techniques de respiration, à un état de conscience modifiée :

De la même manière que la particularité de l'ordre Rufai est de produire un état extatique par le balancement du corps accompagné de la répétition à haute voix du nom de Dieu et de prières; et tout comme les Mevlevi produisent cet «état »en tournant à leur manière au son du ney et de la percussion; de même, les Bektashi disent [...] que la particularité de leur rite ou cérémonie est de produire un état d'intoxication spirituelle par la consommation de vin ou de $\mathrm{rak}^{9}{ }^{9}$ (Birge, $1937: 201$ ).

Pourtant, si technique il y a, l'intoxication éthylique - bien que recherchée n'en épuise pas le sens. Au contraire. Car, dans le même temps que l'on remarque la quantité somme toute considérable d'alcool ingurgitée au cours de la soirée, il est nécessaire d'apprécier l'extraordinaire discipline des participants. Quiconque a à l'esprit les calomnies que la consommation d'alcool fait courir sur le compte des bektaşi ne pourra qu'être étonné par la rigueur qui règne dans le cem. De fait, l'ivresse doit se comprendre au sein d'une discipline, d'un contrôle qui se déploie tant en amont qu'en aval de la consommation. En amont joue le contrôle évoqué précédemment, celui qui est du ressort du saki, et qui est autant affaire de dosage que de rétention. La temporalité propre de l'ivresse est ici une temporalité segmentée, imposée, et la ponctuation du baba est tout autant frustration du buveur, césures dans le flot habituel du boire ${ }^{10}$. En aval s'exerce le contrôle d'un autre officiant, le gözcü - «le vigile », " celui qui voit » - dont le rôle, tout au long de la cérémonie, est, littéralement, de surveiller et punir. Officiant le plus respecté après le baba (en face duquel il est assis), le gözcü doit assurer la bonne tenue du rituel. L'ensemble de la cérémonie est placé sous son regard, et tout dérapage, tout comportement inadéquat, sera immédiatement réprimandé ou sanctionné.

\section{Discipliner le corps}

L'office du gözcü amène à évoquer les règles strictes qui régissent, durant toute la cérémonie, la conduite des participants : ne pas parler, ne pas se déplacer,

9. Ettore Rossi livrera quelques années plus tard une analyse similaire : « L'une des caractéristiques des réunions est l'usage souvent immodéré de l'eau-de-vie (raki). On dit que le raki est indispensable comme stimulant de l'imagination et de l'esprit, et qu'il est apte à favoriser l'état de grâce des assemblées mystiques : autre signe de l'atmosphère de fascination et d'exaltation affectée qui domine les rites des Bektashi »(Rossi, 1943 : 74).

10. Imposée dans sa temporalité, l'ivresse n'en est pas pour autant forcée, et chacun peut refuser de boire. 
ne pas étirer ses jambes, ne pas sortir de la pièce sans autorisation expresse du gözcü, etc. La cérémonie tout entière est prise dans un système de sanction (ceza), de répression : gestes ou paroles déplacés, erreur dans le déroulement des offices, manquement à la hiérarchie seront réprimandés comme marque d'un moi trop encombrant (benlik) et dûment sanctionnés. Si un manquement bénin est réprimandé oralement (quelques fois un regard du gözcü suffit), un dérapage plus grave est sanctionné par une amende, voire par une exclusion temporaire de la cérémonie. L'exclusion définitive (düşkünlük), sanction la plus lourde de ce système de répression, est réservée à une faute commise hors de la cérémonie ${ }^{11}$ (principalement le divorce ou l'adultère).

L'attention et, le cas échéant, la répression, s'attachent plus spécifiquement aux corps des initiés, des corps engoncés dans une stricte discipline, la maîtrise du corps étant par ailleurs, sur le mode de l'injonction, l'un des mottos de la confrérie : "Sois maître de tes mains, de ta langue et de tes reins " (eline, diline, beline sahip ol). Toute une machinerie corporelle se déploie au cours du cem; elle est fondée sur deux " ritournelles gestuelles » qui traversent le rituel : le niyaz ( "supplication ») et le dar (pour darağacı, " le gibet»). Le niyaz est un geste de révérence qui consiste à embrasser à distance; le dar marque la contrition et s'effectue debout, les deux pieds accolés, un orteil sur l'autre, les mains croisées sur la poitrine, le buste penché en avant. Plus trivialement, on évoquera cette position assise les jambes repliées, que l'on pourrait croire habituelle pour les participants si un officiant, accordant à l'occasion permission de "s'asseoir confortablement » ne venait pas confirmer l'inconfort du corps. Il n'est d'ailleurs pas inutile de préciser la durée de ces cérémonies qui s'étirent sur presque sept heures les jours fastes; pas inutile non plus de mentionner l'ennui qui peut saisir le visiteur, et ce temps long est certainement un autre procédé du travail du corps.

Cette retenue frappe d'autant plus que sont réunis là tous les éléments qui évoquent immanquablement en Turquie la licence ou la jouissance : le lourd plateau chargé de nourriture, la viande de mouton, l'alcool, la musique, la mixité sexuelle, la danse... Mais, de la même manière que l'alcool se transforme en dem, chacun de ces éléments change au moins nominalement de nature dans le cadre rituel : les chants deviennent "respiration" (nefes), le grand plateau de nourriture une "bouchée " (lokma), les danses une "écoute" (semah) - et le baba précisera durant son sermon que "ceci n'est pas une danse » - tandis que les participants sont hélés du nom d' "âmes » (canlar) ou, plus éloquent encore, de «parfaits» (erenler).

11. Jérôme Cler décrit ailleurs l'amende infligée aux officiants-musiciens après qu'ils eurent manqué leur entrée dans le déroulement du rituel (Cler, 2013 : 225). 


\section{Émousser l'ego}

L'ivresse entretient une dialectique évidente avec ce vaste système de contrôle du corps. Ainsi en est-il par exemple de la "danse des Quarante ", danse tout en retenue, presque en équilibre, sans aucun contact, exigeant une légère rotation en passant devant les dignitaires (pour ne pas leur tourner le dos), et qui n'accuse ni ne tolère aucune marque d'extase ou d'ivresse, quand bien même est-elle dansée à la fin du muhabbet, soit au pic de l'ivresse collective.

Deux logiques contradictoires sont en jeu :

- d'une part l'extase, ou toute manifestation publique d'une conscience altérée, est hors de question. Cela n'est pas recherché ici, et serait immédiatement endigué ;

- d'autre part, la consommation, bien que strictement contrôlée, est loin d'être uniquement symbolique. Durant les deux bonnes heures que dure le muhabbet, les participants ingurgitent suffisamment d'alcool pour sérieusement enivrer les plus aguerris des buveurs.

Mais c'est précisément dans la mise en tension de ces deux logiques, institution de l'ivresse et discipline du corps, que se dessine l'usage initiatique du dem. L'alcool tient ici de l'injonction paradoxale ${ }^{12}$ : sois ivre mais reste maître de tes actes « de tes mains, de ta langue et de tes reins ». De cette injonction paradoxale, une des autorités du village donne le sens en ces termes : "L'alcool est la clé de l'homme" (dem insanm anahtaridır). Ce n'est pas autre chose qu'a exprimé le poète Hatayi ${ }^{13}$, l'un des plus chantés durant les cérémonies, par ce vers d'un abord surprenant : "J'ai bu la coupe, j'ai repris conscience » (İçmişem bir dolu olmuşum ayık). L'ivresse est ici pensée comme révélateur d'une intériorité, la mesure d'un « ego » $(n e f s)$ qui se trouve mis en procès et sur qui s'exerce pleinement le système répressif. Il y aurait beaucoup à dire sur cette notion de nefs, notion coranique complexe (Blachère, 1948) autour de laquelle tourne une bonne partie de l'enseignement confrérique (Böwering, 1996 ; Silverstein, 2007), et que l'on pourrait définir par opposition à l' " âme immortelle » (rub) comme l' "âme charnelle ", le siège du désir et donc la source des possibles vices. Or l'initié, selon les dires des intéressés, n’est autre que celui qui a "émoussé son ego »

12. Même si j'entends ici le mot dans un sens large et commun, on notera que Gregory Bateson, qui développa la notion, rédigea un article sur l'alcoolisme ( La cybernétique du "soi" : une théorie de l'alcoolisme ", in Vers une écologie de l'esprit 1, Éditions du Seuil, 1977). Une réflexion qui porte sur le "groupe de prémisses qui sous-tendent le concept occidental de soi (self) » (p. 230), évoque le fait de « mettre à l'épreuve la maitrise de soi » (p. 243), le «therapeutic double bind" (p. 243), l'idée que la " panique [de l'alcoolique] est due à la découverte que ça (c'est-à-dire le système : soi-même plus le véhicule) le dépasse. »(p. 246). Les buveurs initiés ne sont certes pas alcooliques, mais ce ça (en italique dans le texte) est peut-être une autre définition possible du nefs.

13. Nom de plume, Hatayi n'est autre que Chah Ismaël, le fondateur de la dynastie des safavides qui régna sur l'Iran pendant plus de deux siècles (1501-1736). 
(nefsini körletmek), qui l'a purgé du vice (kötülük) et du moi (benlik); ce n'est d'ailleurs qu'à ce prix qu'il peut siéger dans le cem, toute expression contraire entraînant irrémédiablement son exclusion. Qu'à travers le corps ivre s'exprime quelque chose de l'essence de l'homme, de sa personne, place de facto la consommation rituelle d'alcool dans le registre de l'épreuve, de l'exercice spirituel - à la fois confrontation et discipline du moi - visant à s'assurer de la perfection des participants, ceux-là mêmes qui s'interpellent du nom d' "âmes " (canlar) ou de «parfaits» (erenler).

L'on voit ainsi comment la transgression d'un précepte islamique est résorbée : l'alcool n'est plus objet d'un interdit, comme cela est le cas dans l'islam sunnite, mais le moyen d'une mise à l'épreuve des initiés, le contrôle ne s'exerçant plus sur la boisson mais sur le corps de l'initié. Pour le dire autrement, la discipline de l'ivresse se fonde sur un déplacement d'ordre praxique : non plus un contrôle de l'alcool, mais un contrôle par l'alcool.

\section{La loi et la voie}

Cette " épreuve », et ce déplacement, doivent se comprendre dans une logique propre à l'islam confrérique, qui professe une distinction entre deux niveaux de compréhension du message islamique, deux niveaux de lecture du Coran :

- une lecture exotérique (zahir), littérale, qui est celle de la charia (şeriat), la loi islamique ;

- et une lecture ésotérique (batın) ${ }^{14}$, que l'on dit couramment "mystique » ou "soufie", et qui est celle de la voie confrérique : la tarikat ${ }^{15}$.

S'opère ainsi, pour les initiés, une divergence entre la loi et la voie, entre la loi islamique qui s'applique à la majorité et n'est que respect de la règle, et la voie confrérique qui est suivie par les initiés et consiste en un enseignement initiatique, une recherche de la Vérité (Hakk). John Kingsley Birge décrit en détail cette riche gnose initiatique et son argumentation qui associe Muhammed à la loi et Ali à la voie (Birge, 1937 : 106), la dialectique du montré et du caché, de la dualité apparente et de l'unicité réelle de l'être. Il explique également comment la voie se donne comme une quête de perfection humaine - vers cet " homme parfait » (insan-i kamil) évoqué par Ibn Arabi - qui ne peut s'entreprendre que sous l'égide d'un maître spirituel. Insistant sur le mystère, il note enfin que, détenteur d'un sens caché, l'initié à la voie confrérique n'est plus tenu aux prescriptions de la charia (Birge, 1937 : 107). De fait, l'enseignement

14. "Zâhîr et bâtın entretiennent les mêmes rapports que l'amande et sa coque, disent les Bâtınî : “celui qui trouve une amande n'attache pas d'importance à sa coque”. Il en est de même du Coran, dont il convient d'abord de décrypter le sens caché pour atteindre la Vérité » (Gökalp, $1980: 175)$.

15. On remplace à l'occasion ce mot arabe par son équivalent turc : yol, «le chemin ». 
ésotérique procède par écarts avec le sens commun, mais selon un écart qui n'est jamais rupture et vise au contraire à renverser la compréhension de la règle en lui suppléant un autre niveau de sens.

Ce travail de reformulation s'apprécie pleinement dans la mise en regard de la sentence citée précédemment - "l'alcool est la clé de l'homme » - et d'un proverbe attribué au prophète Muhammed, stipulant que «l'alcool est la mère de tous les vices » (içki tüm kötülüklerin anasıdır). Malgré l'apparente divergence entre les deux formules, elles n'entretiennent aucune contradiction formelle. La déclaration bektaşi ne vient pas nier la sanction du prophète mais la mettre en perspective, presque l'expliciter selon une logique toute didactique que l'on résumerait ainsi : si l'alcool est la mère de tous les vices, c'est en tant qu'il révèle les vices inhérents à l'homme, et ce n'est que parce que l'homme possède des vices que l'alcool doit lui être interdit. De sorte qu'il n'y a dès lors aucun inconvénient à ce que boivent ceux qui ont surmonté leurs propres vices. On voit comment le commentaire confrérique, en déployant un espace interprétatif, vient détourner le sens premier, selon un principe d'exégèse qui n'est pas sans évoquer la mystique chiite (Amir-Moezzi et Jambet, 2014).

La position de la confrérie vis-à-vis de l'alcool semble toute entière ramassée dans un poème du derviche bektaşi stambouliote Edip Harabi (1853-1917), que je citerai dans son intégralité tant il est éloquent pour notre propos :

Ô ascète ne méprise pas le vin

Sois un homme ici-bas, ce monde est périssable

Licite à l'averti, illicite au non-averti

Nous nous buvons, nous sommes irréprochables

C'est par piété que nous buvons

Ne pas boire est un tourment

Ton esprit ne peut saisir cela

Nous, c'est à la taverne que nous avons trouvé la perfection

Nous sommes chandelle les Nuits des Chandelles ${ }^{16}$

Nous sommes la mèche dans la chandelle

Nous sommes preuve vers la Vérité

Mais les aveugles ne peuvent voir cela

À toi l'impie, le vin est prohibé

Pour boire, tu attendras l'autre monde

Ne querelle pas Harabi pour cela

Il ne connait ni licite ni illicite ${ }^{17}$

16. Référence à la tradition ottomane d'illuminer les mosquées de chandelles pour certaines fêtes religieuses.

17. Ey zahit şaraba eyle ihtiram/Insan ol cihanda bu dünya fani/Ehline helaldir na-ehle haram/Biz içeriz bize vebali yoktur // Sevap almak için içeriz şarap/İçmezsek oluruz düçar-ı azap/Senin aklin ermez bu başka hesap/Meyhanede bulduk biz bu kemali // Kandil geceleri kandil oluruz/Kandilin içinde fitil oluruz/Hakkı göstermeye delil oluruz/Fakat kör olanlar görmez bu halı // Sen münkinsin sana haramdır bade/Bekle ki içesin öbür dünyada/bahs açma Harabi bundan ziyade/Çünkü bilmez haram ile helali. 
«Licite (helal) à l'averti, illicite (haram) au non-averti » : se trouve ici exprimée avec une remarquable clarté l'ambivalence attribuée à la notion d'interdit, d'illicite (haram). Cette borne de la morale musulmane se transforme en notion relative, relationnelle, ce qui est tout à la fois scandaleux et étymologiquement approprié, puisque haram signifie l'interdit mais également le sacré. Quant à la suite du poème, elle est d'une autre teneur. Et si l'auteur et ses compagnons peuvent boire parce qu'ils sont "irréprochables ", leur contradicteur est d'abord un idiot ( "Ton esprit ne peut saisir cela ») avant d'être un "impie ». Edip Harabi est certes réputé pour son sens de la diatribe, comme d'autres poètes célèbres de la confrérie ${ }^{18}$, mais s'énonce dans ces quatrains un discours à visée sociale qu'il s'agit de prendre au sérieux. Ces vers ne se contentent pas de justifier une pratique, de l'expliciter ; ils viennent prendre à partie un opposant, traçant - par le biais de l'alcool - une ligne de démarcation entre un « toi » et un « nous ».

\section{Structurer un ordre}

J'ai évoqué en commençant, sans trop m'y attarder, les calomnies dont a fait l'objet la confrérie, calomnies nourries bien sûr par le secret de la cérémonie et la mixité sexuelle, mais qui s'attachent tout particulièrement à la consommation d'alcool. Or, une fois l'ambivalence de l'interdit confirmée, ce ne sont plus ceux qui boivent qui sont frappés de soupçon, mais bien ceux qui ne boivent pas : puisque l'alcool révèle le vice, ne pas boire provoque les soupçons. M'a ainsi été rapportée une histoire vécue qui ressemble étrangement à ces anecdotes humoristiques qui courent sur le compte des bektaşi (Balivet, 2014) : un bektaşi de Thrace grecque rencontra à Ankara un haut dignitaire de l'ordre qui lui offrit du vin. Le visiteur, gêné, refusa en expliquant qu'il ne buvait pas, ce qui lui valut la réponse suivante: "Si tu ne bois pas, c'est ou que tu n'as pas confiance en moi, ou que tu n'as pas confiance en toi ${ }^{19}$ ". C'est, à peu de choses près, le même discours que tient un dignitaire de Tekke Köyü à propos des sunnites qui critiquent leur penchant pour l'alcool : si eux ne boivent pas, explique-t-il, "c'est qu'ils ne se maîtrisent pas, qu'ils ont peur d'eux-mêmes " (kendilerinden korkuyorlar, kendilerine sahip olamadilar). De sorte que le déplacement praxique évoqué plus haut s'accompagne d'un renversement du stigmate, contre-discours dont le principal ressort est l'hypocrisie supposée de leurs détracteurs, à la fois en ce qu'ils boiraient malgré tout en cachette, et en ce qu'ils proscriraient sur terre ce qui est promis au Paradis. C'est en ce sens qu'il faut entendre ce vers de Harabi : "Pour boire, tu attendras l'autre monde". Ce renversement ne tient pas uniquement du "c'est celui qui le dit qui l'est », il participe activement de la légitimation de l'ordre confrérique, il est au sens plein un positionnement social qui se déploie depuis le rituel. Arguant du contrôle étroit des fidèles exercé durant la cérémonie du cem, l'une des autorités de Tekke Köyü explique ainsi

18. Kaygusuz Abdal et Neyzen Tevfik, pour ne citer que les plus connus.

19. Giorgos Mavromatis, communication personnelle. 
que : «Tout le monde peut aller à la mosquée, l'on peut être un voleur ou un criminel et aller à la prière ; alors que dans le cem, celui qui a commis une faute sera exclu et ne pourra participer à la cérémonie ${ }^{20} »$. On retrouve là le principe qui présidait à l'articulation des deux sentences sur la nature de l'alcool : non pas une rupture, mais un renversement par l'adjonction d'un nouveau niveau de sens. Renversement qui vise implicitement à affirmer que « Le bektachisme n'est rien d'autre que le vrai islam ${ }^{21}$ ".

Au sein de ce régime relationnel affirmant la prééminence des initiés et de la confrérie, la consommation d'alcool assume le rôle d'administration de la preuve, rouage essentiel de l'articulation de l'ordre confrérique. De fait, elle vient à la fois attester, par l'ivresse, de la perfection des initiés, et mettre à distance le commun par la transgression volontaire de l'interdit qui frappe l'alcool. Entre l'un et l'autre, entre l'ivresse-technique du corps et l'alcool-stratégie sociale (Houseman, Severi, 2009 : 219), il importe, pour paraphraser Jean-Pierre Warnier, « de souligner une distinction entre l'artefact pris pour sa valeur praxique dans un système d'action et l'artefact pris pour sa valeur signe dans un système de communication ou de connotation ${ }^{22}$ ». Or, dans notre cas, la connotation est tout sauf anodine. Réduire l'alcool à ses effets corporels, réduire l'alcool à l'ivresse, serait résorber à peu de frais l'immense charge subversive qu'il comporte en contexte musulman. Et s'il est ce qui donne corps à une pensée, il est surtout ce qui sépare irrémédiablement du reste de la communauté des croyants, celle qui vit dans les villages alentour. Son usage dans la cérémonie est éminemment problématique : il est ce qui est publiquement indicible et, in fine, injustifiable. Ne nous y trompons pas, le « discours » confrérique évoqué précédemment ne peut prétendre à une quelconque portée dans l'espace public - il y serait proprement inaudible - et reste confiné à l'entendement des initiés. Tout au contraire, la position publique de la confrérie consiste à cacher, à minorer, à insister sur le respect de la charia (Birge, 1937 : 107 ; Soileau, 2014), ou encore à se placer sur le registre métaphorique, en jouant sur l'ambiguïté du mot dem qui ne désigne pas explicitement l'alcool. Autrement dit, à désamorcer ce qui s'apparente à une transgression impardonnable. À Tekke Köyü même, des crispations ont pu survenir quant à l'usage de l'alcool, et, pour un temps, un groupe, que les autres nommèrent par dérision "les buveurs de thé " (çaycılar), décida de remplacer le rakı par des

20. Isabelle Rivoal témoigne d'une position similaire chez les Druzes vis-à-vis de l'islam, et cite l'un de ses informateurs : " Après avoir tué, il suffit à un Musulman de tourner trois fois autour d'une pierre noire (allusion au sanctuaire de la Kaaba à La Mecque) pour être purifié et se voir autoriser l'entrée dans une mosquée. Chez nous, cela n'est pas possible, on regarde le comportement, l'intention (niyya), et celui qui a tué ne pourra jamais entrer dans la religion. La religion druze est plus difficile" (Rivoal, $2000: 110$ ).

21. Propos d'un baba bektaşi albanais in Clayer (1990:77-78) ; l'enjeu n'est autre que la définition de ce "vrai islam".

22. «Il m'importe ici de souligner une distinction qui me semble essentielle entre l'artefact pris pour sa valeur-signe dans un système de communication ou de connotation (comme l'ont illustré Barthes, Lévi-Strauss, Baudrillard et de nombreux autres analystes), et l'artefact pris pour sa valeur praxique dans le mouvement et dans un système d'action (comme c'est le cas chez Berthoz, Petit, Rizzolatti, Foucault, etc.) "(Warnier, 2009 : 18). 
infusions ${ }^{23}$. Quant à la cérémonie publique organisée chaque année lors des célébrations du saint local, elle se passe de cette pratique. Cet « assèchement de l'ivresse » dans l'espace public est évident dans les communautés alevi ${ }^{24}$, dont certaines $^{25}$ partagent avec les bektaşi la pratique du dem mais qui, lors des cérémonies publiques dans le cadre d'associations urbaines, ont également généralisé l'usage du thé.

La pression est forte et le choix est simple : la dissimulation ou le thé. La consommation rituelle d'alcool se trouve inextricablement liée au secret ${ }^{26}$ (Zarcone, 2002 : 54), parce qu'inacceptable hors de l'enseignement ésotérique. Et n'a pas vocation à l'être ${ }^{27}$. De sorte que si, au cours de ces cérémonies, comme dans de nombreuses sociétés, l'alcool vaut avant tout par ce qu'il fait advenir (ici le $n e f s$ ), la présence d'alcool, son usage, enjoint en contexte musulman une dissimulation au monde extérieur, un retrait, qui est également discipline sociale. Jeu de structure, le dem procède à la fois du dévoilement et du voilement. S'élabore ainsi un double réseau de relations : une disjonction par l'alcool d'avec les sunnites, et une initiation par l'ivresse sous la gouverne du baba. Ce n'est qu'en prenant la pleine mesure de ce double réseau et de son articulation qu'il convient de parler d'un usage initiatique du dem, d'un boire rituel. Le dem est à la fois l'expérience d'un "dedans » par l'ivresse et la mise à distance d'un « dehors » par l'alcool, selon une dialectique de l'ésotérisme musulman à l'élaboration de laquelle il participe puissamment. Et cette dialectique, réseau structuré de relations, induit une hiérarchie, ou, à proprement parler, un ordre. Pour le dire autrement, l'expérience du dem suffit ici à déployer un espace ésotérique divergent du monde social, où vient se distinguer et se structurer l'ordre confrérique.

\section{Nicolas ELIAS \\ Musée du Quai Branly \\ Centre d'études turques, ottomanes, balkaniques et centrasiatiques ruesaintlazare@hotmail.com}

23. Je rappelle que le mot dem est employé également, et plus couramment, pour le thé fortement infusé qui sera coupé à l'eau bouillante.

24. Alevi désigne un groupe confessionnel peu homogène. Historiquement proches de la confrérie bektaşi, avec laquelle elles partagent leurs figures tutélaires et un certain nombre de pratiques, les communautés alevi suivent une structuration sociale singulièrement différente (hérédité de l'appartenance et organisation des congrégations autour de lignages sacrés).

25. "Alors que l'alcool est courant chez les bektachis et dans les cem [alevi] des régions méditerranéenne et égéenne, il est formellement interdit dans nombre de cérémonies d'Anatolie centrale et orientale " (Massicard, $2005: 151$ ).

26. «Par exemple, le fait de boire ou de ne pas respecter la séparation des sexes durant les séances de prières est, pour les bektachis, lié au secret " (Zarcone, 2002: 54) ; "The fact that the Bektashis used wine and raki has been generally known in Turkey perhaps during their whole history. The meaning of their use of it, however, as a Bektashi Baba has assured me is "their secret" " (Birge, 1937: 159).

27. "Si le sens caché des versets abolissait le sens apparent, si la vérité rendait vaine la sharî'a, ou religion du culte, il s'ensuivrait que la religion intérieure deviendrait, ipso facto, religion extérieure, et menacerait de devenir une domination et l'exercice d'un pouvoir temporel. La haqîqa se renverserait en sharî’a nouvelle et arbitraire» (Amir-Moezzi et Jambet, $2014: 145$ ). 


\section{Bibliographie}

Amir-Moezzi Mohammad Ali, Jambet Christian, 2014, Qu'est-ce que le shî̀isme? Le Cerf.

Balivet Michel, 2014, Les Dits du derviche Bektachi, Éditions Non Lieu.

BATESON Gregory, 1997, "La cybernétique du "soi": une théorie de l'alcoolisme ", in Vers une écologie de l'esprit 1 (Steps toward an ecology of mind, 1972), Éditions du Seuil.

BIRGE John Kingsley, 1937, The Bektashi order of dervishes, Hartford Seminary Press.

BlachÈRe Régis, 1948, "Note sur le substantif nafs "souffle vital”, "âme”, dans le Coran ", Semitica, 1, p. 69-77.

BöWERING Gerhard, 1996, "Règles et rituels soufis ", in Popovic A. et Veinstein E. (éds.), Les Voies d'Allah. Les ordres mystiques dans le monde musulman des origines à aujourd'hui, Librairie Arthème Fayard, p. 139-156.

Clayer Nathalie, 1990, L'Albanie, pays de derviches. Les ordres mystiques musulmans en Albanie à l'époque post-ottomane (1912-1967), Osteuropa-Institut der Freien Universität Berlin.

Cler Jérôme, 2013, « Neden bu ikilik ? Pourquoi cette dualité ? Ethnographie de la division dans un village bektashi du Taurus ", in Clayer N., Papas A. et Fliche B. (éds.), L'autorité religieuse et ses limites en terres d'Islam, Brill.

GÖKALP Altan, 1980, Têtes rouges et bouches noires. Une confrérie tribale de l'Ouest anatolien, Société d'ethnographie.

Houseman Michael, Severi Carlo, 2009, Naven ou le donner à voir. Essai d'interprétation de l'action rituelle, Éditions du Centre national de la recherche scientifique - Éditions de la Maison des Sciences de l'Homme.

KöPrÜlü Fuat, 2001, «Abdal Musa » (traduit du turc et annoté par Catherine Pinguet), Journal of the History of Soufism, 3, p. 325-347.

MASSICARD Élise, 2005, L'autre Turquie. Le mouvement aléviste et ses territoires, Presses universitaires de France.

MéLIKofF Irène, 1998, Hadji Bektach : un mythe et ses avatars. Genèse et évolution du soufisme populaire en Turquie, Brill.

RivoAL Isabelle, 2000, Les maîtres du secret. Ordre mondain et ordre religieux dans la communauté Druze en Israël, Éditions de l'École des hautes études en sciences sociales.

Rossi Ettore, 1943, "Credenze ed usi dei Bektashi », Studi e materiali di storia delle religioni, 18, p. 60-80.

SiLverstein Brian, 2007, "Sufism and Modernity in Turkey: From the Authenticity of Experience to the Practice of Discipline ", in Bruinessen M. van et Day Howell J. (éds.), Sufism and the Modern in Islam, I. B. Tauris, p. 39-60.

SOILEAU Mark, 2012, "Spreading the sofra: Sharing and partaking in the bektashi ritual meal ", History of religions $\mathrm{n}^{\circ}$ 52, p. 1-30.

-, 2014, "Conforming Haji Bektash: A saint and his followers between orthopraxy and heteropraxy ", Die Welt des Islams, 54, p. 423-459.

WARNIER Jean-Pierre, 2009, "Les technologies du sujet", Techniques et cultures [en ligne], 52-53.

ZARCONE Thierry, 2002, Secret et sociétés secrètes en Islam. Turquie, Iran et Asie centrale. XIX-XX ${ }^{e}$ siècles, Archè. 


\section{La discipline de l'ivresse dans une confrérie musulmane de Turquie}

Quel sens (et quels effets) peut avoir la consommation rituelle d'alcool au sein d'une confrérie qui se réclame explicitement de l'islam ? C'est à cette question que s'attache ce texte, à partir d'une enquête dans un village de la Turquie méridionale affilié à la confrérie bektaşi. Là, lors des rituels du cem, de l'alcool est consommé en quantité conséquente. Mais l'ivresse ainsi instituée doit se comprendre en relation avec une éthique de la maîtrise du corps - "de la main, de la langue et des reins " - et relève dès lors de l'épreuve spirituelle. De sorte que l'alcool n'y est plus l'objet d'un contrôle social mais le moyen d'une discipline de soi. Et joue un rôle majeur dans la structuration de l'ordre confrérique.

Mots-clés : rituel, religion, islam, alcool, discipline, Turquie.

\section{The discipline of drunkenness in a Muslim brotherhood of Turkey}

What could be the meaning (and effects) of alcohol drinking in ritual context within a Muslim brotherhood? Being based on fieldwork in a Turkish village affiliated with the bektassi brotherhood, this paper aims at answering that question. During the cem rituals that take place in the village, alcohol is consumed in large quantities. But the induced drunkenness shall be understood in relation to a strong ethic of bodily control - "master your hand, your tongue and your waist" being the brotherhood's motto -, it thus operates as a spiritual trial. From that perspective, alcohol is no more the object of social control but the mean of self-discipline, and it plays a major role in the shaping of the brotherhood's symbolic order.

Key words: ritual, religion, Islam, alcohol, discipline, Turkey.

\section{La disciplina de la embriaguez en una cofradía musulmana de Turquía}

¿Qué sentido (y qué efectos) puede tener el consumo ritual de alcohol en una cofradía que se reivindica explicitamente del islam? Vemos esto en la cofradia bektaşi, en un pueblo de la Turquía meridional. Durante los rituales del cem, se consume alcohol sin moderación. Pero la embriaguez así instituida debe ser entendida en relación con una ética del dominio del cuerpo - "de la mano, de la lengua y de los riñones"-, $y$ da cuenta en ese sentido de la prueba espiritual, de manera que el alcohol no es ya el objeto de una regulación social sino el instrumento de una disciplina de sí. La bebida juega un rol mayor en la estructuración del orden en la cofradía.

Palabras clave: ritual, religión, islam, alcohol, disciplina, Turquía. 